\title{
Microstructure engineering of materials
}

\author{
B. S. Murty • Gandham Phanikumar
}

Published online: 20 December 2011

(C) Indian Institute of Technology Madras 2011

Microstructure of most technical alloys for an engineering application would involve multiple phases that could differ in their physical properties, configuration, morphology, size, volume fraction etc. While the choice of a material for a specific application could involve relevant functional property, often a secondary property (such as freezing range, toughness, corrosion or creep resistance, biological compatibility etc.) could play an overwhelming role in the successful usage of that material. Designing the microstructure, taking the material and processing constraints into account, is one of the major areas of materials research today.

In the recent past, several novel processing techniques have emerged that have aimed at obtaining a specific microstructure of the material with an intended application. Severe plastic deformation, electromagnetic processing of melts, multi-step thermo-mechanical processing, rapid solidification are some examples of emerging techniques where research is focused on both the materials as well as the processing technique to obtain the designed microstructure. Kinetic constraints such as rate of deformation and rate of cooling imposed during the processing have an important role in the evolution of microstructure. Several new characterization techniques and tools such as orientation imaging, focused ion beam milling and analytical electron microscopy have aided in assessing the efficacy of the engineering approach taken in the design of a microstructure. Advances in the computational modeling of the processing techniques and simulation of

B. S. Murty · G. Phanikumar ( $\square)$

Department of Metallurgical and Materials Engineering, Indian Institute of Technology Madras, Chennai 600036, India

e-mail: gphani@iitm.ac.in

B. S. Murty

e-mail: murty@iitm.ac.in microstructure have opened new avenues in the microstructure engineering.

In this thematic volume, we present a collection of five papers that bring out the microstructure engineering in the current state of the art. Arvind Kumar, Miha Zaloznik and Herve Combeau have presented their work on prediction of equiaxed grain structure and macrosegregation in an industrial steel ingot and have compared their results with experiments. Sumantra Mandal, V. Subramanya Sarma and A. K. Bhadhuri have performed grain boundary engineering in alloy D9 through thermo-mechanical processing and have studied influence of process variables and delved on the aspects of micro-mechanisms in depth. Georg J. Schmitz, Bernd Böttger, Jain Eiken, Markus Apel, Antoine Carré, Alexandre Viardin and Gottfried Laschet have reviewed the current status of the phase-field based simulations of microstructure evolution in technical grade alloys. Bikramjit Basu and Garima Tripathi have presented their work on processing and biological evaluation of porous hydroxyapatite/poly methyl methacrylate hybrid composites. Seshacharyulu Tamirisakandala and Dan Miracle have reviewed the efforts to engineer the microstructure of titanium alloys using small additions of boron as alloying element. The materials covered in these studies are of current interest for the industry and the processes chosen are of high impact. This thematic issue has a fine balance of experimental and computational studies on the topic.

Articles in this theme were contributed by researchers whose groups have contributed significantly to this area for last several years. We hope that readers will find this issue valuable and informative on the theme of microstructure engineering of materials. The ubiquitous presence of microstructure characterization tools in academic departments of varied engineering disciplines indicates that slowly and steadily, the mesoscale information of materials is being studied and engineered for desired performance. 